\title{
Malaria Four-year Epidemiological Trends in Sistan and Baluchistan Province, Iran
}

Faezeh Norouzinezhad ${ }^{1}$, Fatemeh Ghaffari ${ }^{2}$, Ahmad Raeisi $^{3}$, Abbas Norouzinejad $^{4}$, Farzad Kaveh $^{5}$

${ }^{1}$ Faculty Member, MSN, BSc, School of Nursing and Midwifery, School of Nursing and Midwifery, Babol University of Medical Sciences, Babol, Mazandaran, Iran

${ }^{2}$ Assistant Professor in Nursing, Ramsar Nursing Care Research Center, Babol University of Medical Sciences, Mazandaran, Iran

${ }^{3}$ Associate Professor of Epidemiology, National Program Manager for Malaria Control, Ministry of Health and Medical Education, Tehran, Iran

${ }^{4}$ Deputy for Administrative Affairs of the Center for Communicable Disease Control, Ministry of Health and Medical Education, Tehran, Iran

${ }^{5}$ Data Manager and Disease Control Expert in the Center for Communicable Disease Control, Tehran, Iran

\section{Type of article: Original}

\begin{abstract}
Introduction: Malaria is one of the foremost public health concerns in Iran, where more than $90 \%$ of malaria cases are reported in the southern and south-eastern areas of the country.

The aim of this study was to assess the epidemiological trends of malaria over a four-year period in in the Sistan and Baluchistan province in south east of Iran.

Methods: This descriptive epidemiological study examined malaria trends in Sistan and Baluchistan province from 2011 to 2014. The study used data collected in accordance with the Iranian Ministry of Health's malaria control and elimination protocol. This protocol has digitized the data reporting system for malaria, and all information were sent online to the Center of Disease Control in the Ministry of Health. In this manner, information on malaria cases in Sistan and Baluchistan were made available for the researchers to analyze. Descriptive and comparative analyses were conducted using the SPSS version 13.

Results: Annual incidence rates reported in 2011, 2012, 2013, and 2014 showed the prevalence of 89.9, 43.9, 38.3 and 36.6 (per 100,000 persons), respectively. Across all 4 years, the highest numbers of cases were found in persons 16-25 years old and among males. Most of the infected individuals were villagers and workers. In total, $64.8 \%$ of patients were Iranian and $29.5 \%$ were Pakistani. The highest number of cases was diagnosed in the cities of Sarbaz and Chabahar, with 1,742 and 1,707 cases, respectively. The results showed that over the last 4 years, $50.8 \%$ of cases have entered into Iran from foreign countries. The majority of cases involved parasites in the trophozoite stage of the life cycle. In terms of surveillance, passive care was reported in the majority of cases, and vivax malaria had the highest prevalence in comparison with other types.

Conclusion: The findings are showing that the care, control and treatment system applied to Sistan and Baluchistan province has had a positive effect on decreasing the prevalence rate of Malaria disease. Meanwhile, it is recommended to policy makers to provide more health controls for border entries, stop irregular immigration and apply more precise case searches in order to have a complete and on time treatment in a way that the chain of transmission of the disease would be cut. Health education and knowledge-ability promotion programs are better to be set in order to develop self-protection and environment improvement among people.

Keywords: Malaria, Epidemiology, Sistan and Baluchistan, Iran
\end{abstract}

\section{Corresponding author:}

Assistant Professor Dr. Fatemeh Ghaffari, Ramsar Nursing Care Research Center, Babol University of Medical Sciences, Mazandaran, Iran. Postal Code: 4691714141, Tel: +98(11)55225151, E-mail: ghafarifateme@yahoo.com Received: May 23, 2016, Accepted: September 12, 2016, Published: January 2017

iThenticate screening: August 30, 2016, English editing: October 27, 2016, Quality control: November 16, 2016

(C) 2016 The Authors. This is an open access article under the terms of the Creative Commons Attribution-NonCommercialNoDerivs License, which permits use and distribution in any medium, provided the original work is properly cited, the use is non-commercial and no modifications or adaptations are made. 


\section{Introduction}

Malaria is one of the most serious mosquito-borne diseases in the world, particularly in tropical and subtropical regions (1). It is a major threat to global health, resulting in approximately 300-500 million clinical cases and 1-3 million deaths each year, mainly among children (2). About 4-5 million Iranians were infected annually, with mortality rates of $30-40 \%$, before Iran launched its malaria control program which led to many economic and social losses $(3,4)$. This disease has remained as one of the foremost public health concerns in Iran despite following the implementation of malaria control program. More than $90 \%$ of Iranian malaria cases occur in the southern and south-eastern regions having common borders with Pakistan and Afghanistan currently (5). Sistan and Baluchistan province, which shares a long border with Pakistan, accounted for 8,657 cases of malaria; in other words, 53\% of all cases in the country in $2007(6,7)$. Taken together, this province is considered one of the three endemic areas for malaria in Iran. Sistan and Baluchistan requires repeated reviews of malaria epidemiology, because it is geographically located in a place where Plasmodium falciparum is endemic and the province is susceptible to malaria epidemics precipitated by several factors including regional climate diversity (8). An essential component for designing accurate strategies for malaria control, depends on having a precise knowledge of the local epidemiology of the disease (9). The analysis of available malaria data in Sistan and Baluchistan is necessary for gaining needed insights for planning malaria control measures and assessing the impact rate of previous interventions. The current study is an investigation of the recent trends in the number of reported cases of malaria in Sistan and Baluchistan from March 2011 to 2014. Moreover, this study aimed to analyze extant threats and opportunities for controlling the disease. In doing so, the authors have extended the scope beyond the localized epidemiology, and the findings can be applicable to other areas faced with the same problem (10).

\section{Material and Methods}

This descriptive study examined malaria trends in the province of Sistan and Baluchistan between 2011 and 2014, using the data collect for the Ministry of Health's malaria control protocol. In Iran's Malaria Case Notification System, managers and practitioners have reported all the malaria cases referred to Zahedan Medical Sciences University healthcare centers, and those referring to the center of fighting against communicable diseases of healthcare networks. In the malaria case recording and detection protocol, healthcare providers and disease control staff collected the information and took blood samples from malaria-suspected clients. Sample collection was performed in healthcare centers, urban and rural healthcare units, public and private hospitals, and passive tables. In the patio table, people who had fever or early malaria infections were referred to healthcare providers who performed blood tests to detect any parasite carrier. The blood samples were then sent to provincial health department laboratories. The results of sample checks analyses were classified as active or passive and sent online to the Center of Disease Control of the Ministry of Health and Medical Education. The results have been sent daily, within 12 hours after sampling. The variable factors of the study were: gender, age, area of residence, nationality, occupation, city, month of sampling, epidemiological classification of the case, life cycle stage of the malaria parasite, surveillance, parasite species, treatment method, malaria symptoms, early malaria infection within the last 12 months, early malaria infection before the last 12 months, history of blood transfusion, intensity of malaria, treatment results, treatment services, and disease outcomes. The intervention data of centers for disease control and prevention (CDC) programs of Iran's Ministry of Health and Medical Education has been used in this study. The data of the study are extracted from the malaria elimination national protocol gathered data, and all the ethics points have been applied during the recording and reporting process. Descriptive and comparative analyses were conducted using the SPSS version 13.

\section{Results}

The malaria annual incidence rates of 2011-2014 were 89.9, 43.9, 38.3 and 36.6 (per 100,000 persons), respectively. The highest rate of privilege occurred in October (16.7\%) and November (15.7\%). In 2011, 2012 and 2014, most of the malaria cases had been reported in Sarbaz (848,394 and 313) and Chabahar (819,305 and 193). In 2013, Sarbaz was still the $1^{\text {st }}$ in number (390) but the $2^{\text {nd }}$ was Saravan (329). Zabol city (one of the big cities in Sistan and Baluchistan province) became the $3^{\text {rd }}$ in number of malaria cases in 2014 (67), while it wasn't faced with such numbers from 2011-2013 when it was the safest city in the province. Males have formed $77.6 \%$ of all cases. Most of the cases (73.4\%) have been reported among the rural population. In terms of the occupations of patients, $31.7 \%$ were workers, $10.6 \%$ were students, and $14.7 \%$ were housewives. The majority of malaria cases had been reported among Iranians (64.8\%), while $29.5 \%$ were Pakistanis (Table 1). The epidemiological classifications of cases included 4 classes of importation from abroad, local transfer (indigenous), coming from the inside, recurrence (relapse), and transfer from the inside. During the four years of the study, $47.3 \%$ of infections were imported from abroad and $39.6 \%$ were locally transferred. In the majority of cases, the parasite was in the trophozoite life cycle 
stage. In terms of surveillance, the majority of the cases (56.8\%) were passive and $40.0 \%$ were active-routine. In active care, people at risk of malaria were screened in terms of presenting malaria symptoms or having the symptoms in the last month at the time of health officials' house to house visits. The peripheral blood smear of suspected cases of malaria were prepared or checked by Rapid Diagnostic test. From 2011 to 2014, vivax malaria had the highest prevalence rate. The results show that oral and parenteral artesunate were the most-used treatment methods. The highest-risk symptoms were impaired consciousness $(0.4 \%)$, hypertension $(0.4 \%)$, and walking difficulty $(0.3 \%)$. By species, $83.0 \%$ of parasites were $P$. vivax and $13.6 \%$ were $P$. falciparum (Table 2). Urban health centers accounted for $38.4 \%$ of sampled cases, rural health centers accounted for $45.3 \%$, and passive table in $9.7 \%$ of them. Peripheral blood smears were reported as suspected in $82.1 \%$ of feverish cases. The data showed that the incidence of vivax malaria was $1.6 \%$ in less than a year and $2.1 \%$ in more than a year. The results also showed that the history of blood transfusion was positive in $0.3 \%$ of cases. In total, $6.5 \%$ of patients were suffering from complicated and severe malaria. Treatment was successful in $83.0 \%$ of cases. Outpatient services were used in $73.6 \%$ of cases, while $5.9 \%$ of cases required hospitalization. Only two cases of death were reported, and $82.9 \%$ of cases showed recovery. The highest age-specific incidence rates (ASIR) per person among 100,000 women studied in 2012 happened in those aged older than 66 (35.7\%); while the rates for 2011, 2013 and 2014 respectively show $81.1 \%, 35.0 \%$ and $20 \%$ for those aged 56-65. The highest ASIR per 100,000 men from 2012 to 2014 were $127.3 \%$, $106.9 \%$ and $93.44 \%$ respectively, related to those aged 16-25; On the other hand, the highest ASIR is related to 2011 for 56-65 year old participants.

Table 1. Distribution of the frequency of reported cases based on the study variables

\begin{tabular}{|c|c|c|c|c|c|c|c|c|c|}
\hline \multirow[t]{3}{*}{ Variables } & & \multicolumn{8}{|c|}{ Years } \\
\hline & & \multicolumn{2}{|c|}{2011} & \multicolumn{2}{|c|}{2012} & \multicolumn{2}{|c|}{2013} & \multicolumn{2}{|c|}{2014} \\
\hline & & $\mathrm{n}$ & $\%$ & $\mathrm{n}$ & $\%$ & $\mathrm{n}$ & $\%$ & $\mathrm{n}$ & $\%$ \\
\hline \multirow[t]{2}{*}{ Sex } & Male & 1816 & 74.7 & 973 & 80.0 & 864 & 79.5 & 709 & 79.8 \\
\hline & Female & 613 & 25.2 & 243 & 20.0 & 223 & 20.5 & 179 & 20.2 \\
\hline \multirow[t]{4}{*}{ Residence } & Urban & 605 & 24.9 & 293 & 24.1 & 195 & 17.9 & 193 & 21.7 \\
\hline & Rural & 1749 & 71.9 & 915 & 75.2 & 837 & 77.0 & 624 & 70.3 \\
\hline & Traveler & 68 & 2.8 & 7 & .6 & 41 & 3.8 & 57 & 6.4 \\
\hline & Nomadic & 7 & .3 & 1 & .1 & 1 & 1. & 6 & 7. \\
\hline \multirow[t]{3}{*}{ Nationality } & Iranian & 1559 & 64.1 & 797 & 65.5 & 703 & 64.7 & 585 & 65.9 \\
\hline & Pakistani & 687 & 28.2 & 371 & 30.5 & 336 & 30.9 & 266 & 30.0 \\
\hline & Afghani & 183 & 7.5 & 44 & 3.6 & 48 & 4.4 & 36 & 4.1 \\
\hline
\end{tabular}

Table 2. Malaria data in Sistan \& Baluchistan, 2011-2014

\begin{tabular}{|c|c|c|c|c|c|c|c|c|c|}
\hline \multirow[t]{3}{*}{ Variables } & & \multicolumn{8}{|c|}{ Years } \\
\hline & & \multicolumn{2}{|c|}{2011} & \multicolumn{2}{|l|}{2012} & \multicolumn{2}{|c|}{2013} & \multicolumn{2}{|c|}{2014} \\
\hline & & $\mathrm{n}$ & $\%$ & $\mathrm{n}$ & $\%$ & $\mathrm{n}$ & $\%$ & $\mathrm{n}$ & $\%$ \\
\hline \multirow{5}{*}{$\begin{array}{l}\text { Epidemiological } \\
\text { classification of cases }\end{array}$} & Imported from abroad & & & 531 & 43.7 & 609 & 56.0 & 579 & 65.2 \\
\hline & Indigenous & & & 495 & 40.7 & 358 & 32.9 & 230 & 25.9 \\
\hline & Domestically transmitted & & & 174 & 14.3 & 96 & 8.8 & 70 & 7.9 \\
\hline & Relapse & & & 6 & .5 & 1 & .1 & 2 & .2 \\
\hline & Introduced & & & 10 & .8 & 16 & 1.5 & 3 & .3 \\
\hline \multirow{4}{*}{$\begin{array}{l}\text { Lifecycle of the malaria } \\
\text { parasite }\end{array}$} & Gametocytes + Trophozoite & 392 & 16.1 & 129 & 10.6 & 180 & 16.6 & 196 & 22.1 \\
\hline & Trophozoite & 1712 & 70.4 & 899 & 73.9 & 737 & 67.8 & 590 & 66.4 \\
\hline & Schizont & 65 & 2.7 & 50 & 4.1 & 35 & 3.2 & 21 & 2.4 \\
\hline & Gametocytes & 260 & 10.7 & 138 & 11.3 & 122 & 11.2 & 76 & 8.6 \\
\hline \multirow[t]{3}{*}{ Surveillance } & Passive & 1361 & 56.0 & 700 & 57.6 & 637 & 58.6 & 497 & 56.0 \\
\hline & Routine & 975 & 40.1 & 484 & 39.8 & 420 & 38.6 & 370 & 41.7 \\
\hline & Active foci & 93 & 3.8 & 32 & 2.6 & 29 & 2.7 & 21 & 2.4 \\
\hline \multirow[t]{3}{*}{ Parasite species } & Plasmodium vivax & 2002 & 82.3 & 1045 & 85.9 & 834 & 76.7 & 784 & 88.3 \\
\hline & $\begin{array}{l}\text { Mixed (vivax and } \\
\text { Falciparum) }\end{array}$ & 76 & 3.1 & 50 & 4.1 & 51 & 4.7 & 10 & 1.1 \\
\hline & Plasmodium falciparum & 351 & 14.4 & 121 & 10.0 & 197 & 18.1 & 94 & 10.6 \\
\hline
\end{tabular}




\section{Discussion}

The results showed that the incidence rate of malaria has declined over the 4 years of the study. Sargolzaie et al. also found that the number of malaria cases had declined between April 2008 and March 2011 in Sistan and Baluchistan (11). In this study, annual incidence rates of over 4 years were 89.9, 43.9, 38.3 and 36.6 (per 100,000 persons), respectively. Salehi et al. (2010) found that in three consecutive years (2005-2008), the annual incidence rates were 469, 345, and 359 (per 100,000 persons), respectively (10). Although the results of this study showed that the incidence of malaria is declining in Sistan and Baluchistan province, it is still a public health problem in this area for numerous reasons including the number of carriers, carriers' resistance to insecticides, emergence and development of Plasmodium resistance to chloroquine, lack of convenient access to health services, tropical climate, long transmission seasons, and unsustainable human, geographical and ecological conditions (10). On the other hand, the previous epidemiological studies have shown that unlike African or other types of malaria, Iranian malaria is dependent to climatic conditions. It means that this unstable situation may be effective in generating the concern that the precipitation in high risk areas increases the possibility of an outbreak of the disease (3). Sistan and Baluchistan province is bordered by the Oman Sea and the Indian Ocean from the south and has a subtropical climate. Due to appropriate temperature and humidity, transmission cycle of malaria is optimal from April to October in some parts of the province $(3,10)$. The results of this study showed that a significant number of patients were Pakistani. In the study of Sargolzaie et al. (2012), 14.1\% of cases were Pakistani and 8.6\% were Afghan (11). Immigration may create some new foci of the disease in the region. Immigrants from Pakistan and Afghanistan may travel back to their countries of origin, which are known as the most dangerous countries for malaria transfer, and then, return to Sistan and Baluchistan province. According to World Health Organization estimates, 3 million people in Afghanistan and 1 million people in Pakistan are found infected with malaria annually (12). In this study, most cases were reported in men. Gender does not directly or naturally influence over the sensitivity or resistance to malaria, but may influence incidence through occupation and the types of clothing worn. Social activities and working outside home may make men more susceptible to mosquito bites and malaria. Therefore, considering the fact that the risk of getting the disease is equal in both genders, prevention and treatment strategies for both genders should be applied equally (13). The ASIR per 100,000 people has shown that the majority of cases in males occurs in 16-25 year olds. This finding was consistent with the results of other studies $(10,11)$. The results showed that most of the reported cases were workers. Low health literacy and poor economic situations among workers can increase the risk of disease complications and higher disease burdens among them (10). In the present study, the cities of Chabahar and Sarbaz reported the highest number of patients. In the research of Sargolzaie et al. (2012), the highest proportions of patients were reported in Chabahar (31\%), Sarbaz (24.3\%), and Nikshahr (10.2\%), respectively (11). In the study by Salehi et al. (2010), as well, Sarbaz and Nikshahr showed the highest API (10). The results showed that most cases were reported in October (16.7\%) and November (15.7\%). However, in the study by Salehi et al. (2010), most cases of malaria were reported in July (10), while Sargolzaie et al. (2012) found that most cases were reported in September and October (11). From 2011 to 2014, vivax malaria had the highest prevalence. Plasmodium vivax malaria is the most prevalent species in Iran (14). These findings are consistent with other studies (10, 11). Most patients were detected through active surveillance and a small number of patients' blood samples which was referred to malaria laboratories, were diagnosed through passive surveillance methods. Similarly, in the other studies most of the cases were diagnosed through active surveillance (3). These trends indicate a high efficacy of health service providers, health workers and officials in executing malaria elimination programs, treatment, and efficient care systems $(7,15)$. A significant percentage of patients recovered and only two cases of death has been reported in the data of present study, which indicates the existence of effective surveillance, control, and treatment systems in the province.

\section{Conclusions}

The findings are showing that the care, control and treatment system applied to Sistan and Baluchistan province has had a positive effect on decreasing the prevalence rate of Malaria disease. Meanwhile, it is recommended to policy makers, to provide more health controls for border entries, stop irregular immigration and apply more precise case searches in order to have a complete and on time treatment in a way that the chain of transmission of the disease would be cut. Health education and knowledge-ability promotion programs are better to be set in order to develop the self-protection and environment improvement among people. The findings can be useful in policy making, prevention and malaria care programs for local and provincial administrators. Future epidemiological studies can be done on exploring the consequences of malaria removal protocol. 


\section{Acknowledgments:}

The authors sincerely acknowledge their gratitude for the efforts of the Malaria Control Center in the Iranian Ministry of Health and Medical Education. We also appreciate the efforts, precise data, and honesty of the malaria experts at the district health care centers in Sistan and Baluchistan province.

\section{Conflict of Interest:}

There is no conflict of interest to be declared.

Authors' contributions:

All authors contributed to this project and article equally. All authors read and approved the final manuscript.

\section{References:}

1) World Health Organization (WHO). World malaria report 2013. Available from: http://www.who.int/malaria/publications/world_malaria_report_2013/report/en.

2) Hay SI, Okiro EA, Gething PW, Patil AP, Tatem AJ, Guerra CA, et al. Estimating the global clinical burden of Plasmodium falciparum malaria in 2007. PLoS Med. 2010; 7(6): e1000290. doi: 10.1371/journal.pmed.1000290.

3) Motabar M, Azizi F, Janghorbani M, Hatami HE. Control of Common disorders in Iran. Tehran: Khosravi publication; 2003. Journal of Isfahan Medical School Original Article. 29(132): 502-29.

4) Ataei B, Labaf Ghasemi R, Sadri GH, Ramezanpour E, Saeedi E. A survey on malaria in Isfahan Province during years 1986 to 1996 . Trauma Monthly. 2016; 5(1): 63-7.

5) Soleimani-Ahmadi M, Vatandoost H, Zare M, Alizadeh A, Salehi M. Community knowledge and practices regarding malaria and long-lasting insecticidal nets during malaria elimination programme in an endemic area in Iran. Malaria journal. 2014; 13(1): 1-7. doi: 10.1186/1475-2875-13-511.

6) Baserie HR, Holakoei Naeenei K, Raeesie A, Shahandeh Kh, Akbarzadeh K, Ranjbar M, et al. Comparison of knowledge, attitude and performance of Afghan refugees and Iranians to prevent transmission of malaria in the city of Iranshahr. Iranian journal of epidemiology. 2007; 3(3-4): 7-13.

7) Jadgal KM, Zareban I, Rakhshani F, Shahrakipour M, Sepehrvand B, Alizadeh Sivaki H. The effect of health education according to the theory of planned behavior on malaria preventive behavior in rural men of Chabahar. J Research Health. 2012; 2(2): 237-46.

8) Salehi M, Mohammad K, Farahani MM, Zeraati H, Nourijelyani K, Zayeri F. Spatial modeling of malaria incidence rates in Sistan and Baluchistan province, Islamic Republic of Iran. Saudi Med J. 2008; 29(12): 1791-6. PMID: 19082235.

9) Najera JA. Epidemiology in the strategies for malaria control. Parassitologia. 2000; 42(1-2): 9-24. PMID: 11234336.

10) Amirmajdi MM, Mashhadi IE, Hakemi Y, Mashhadi AE, Mirinezhad A. Analysis of malaria epidemic features in Sistan and Baluchistan province, southeast of Iran, 2005-2008. Iranian Red Crescent Medical Journal. 2010; 12(3): 247-53.

11) Sargolzaie N, Salehi M, Kiani M, Sakeni M, Hasanzehi A. Malaria Epidemiology in Sistan and Balouchestan Province during April 2008-March 2011, Iran. ZJRMS. 2014; 16(4): 41-3.

12) Soleimanifard S, Akbari M, Sabetghadam M, Saberi S. Malaria Situation in Isfahan in the Last Five Years. Journal of Isfahan Medical School. 2011;29(132).

13) Edrissian G. Malaria in Iran: Past and present situation. Iranian Journal of Parasitology. 2006; 1(1): 1-14.

14) Manouchehri AV, Zaim M, Emadi AM. A review of malaria in Iran, 1975-90. J Am Mosq Control Assoc. 1992; 8(4): 381-5. PMID: 1474383.

15) Raeisi A, Nikpoor F, Ranjbar Kahkha M, Faraji L. The trend of Malaria in IR Iran from 2002 to 2007. Hakim Health Sys Res. 2009, 12(1): 35-41. 